\title{
Hypertension Prevention and Control: Effects of a Community Health Nurse-led Intervention
}

\author{
Osuala Eunice $\mathbf{O}^{*}$ \\ Department of Nursing Science, Nnamdi Azikiwe University, Nnewi Campus, South East, Nigeria
}

\begin{abstract}
Hypertension is a major risk factor for Cardiovascular Disease (CVD) with complications such as stroke and heart failure. Knowledge and attitude about hypertension have been indicated to influence practice of healthy lifestyle which has implications for hypertension prevention and control. There are anecdotal reports of sudden death and stroke in Isunjaba. However, there is no documentation about their lifestyle practices relating to hypertension. Health information given by nurses may positively influence healthy behaviours such as exercise, weight control, appropriate nutrition and regular Blood Pressure (BP) checks. This study was designed to assess the effects of a Community Health Nursing Intervention $(\mathrm{CHNI})$ on knowledge, attitude and lifestyles relating to hypertension among residents of Isunjaba, Imo State, having the economic advantage of population-focus study in mind. There was significant difference in knowledge, attitude, and lifestyle of the two groups after intervention, $\mathrm{P}$ value $<0.05$. Health Education about hypertension to improve knowledge, attitude as well as positive lifestyles among populations should be supported by nurses, agencies and the Government.
\end{abstract}

Keywords: Blood pressure-check; Health behaviours; Populationfocus; Nurse-led intervention; Lifestyle-modification

\section{Introduction}

The concern over cardiovascular disease is especially relevant in the healthcare of a developing nation like Nigeria. In Nigeria, 57 million people are estimated to be hypertensive with many still undiagnosed. Hypertension is a disease that is both common in urban and rural populace $[1,2]$. It has been linked to unhealthy lifestyles habits (lack of exercise and rest, consumption of alcohol, tobacco products, excess fatty foods and dietary sodium) which are common in our society today [3]. According to Das either due to poverty or affluence our lifestyle (nutrition pattern, social habits and working culture) has changed and thus increased the predisposition to hypertension [4]. Hypertension is identified as the leading cause of Target Organ Damage (TOD) like blindness, kidney failure and coronary artery diseases. Emerging data from hospital studies show that hypertension or its complication is the most common non-communicable disease in Nigeria [5].

By casual observation cases of stroke and sudden deaths have been noted in Isunjaba in Isu Local Government Area of Imo State by the researcher. These cases may be linked to cardiovascular diseases (CVD). The world being a global village, Isunjaba need to be protected from cardiovascular diseases (CVD). Hypertension if left unchecked especially in the rural area where the population is predominantly that of old people, would increase its incidence, cases of stroke, heart failure, glaucoma and renal failure. Even though many studies had been conducted on hypertension in various continents as Asia, America, and Africa by researchers [6-8]; only a few had been done in the rural area of Nigeria [9-13]. Isunjaba of Isu Local Government Area in Imo State being a rural community is not excluded.

Knowledge of risk factors, symptoms, prevention, management, and complications of hypertension may be lacking in rural dwellers in this community. The community blood pressure and attitude to hypertension prevention as well as lifestyle habits of the people is not known as few studies have been done on hypertension among rural dwellers in Nigeria. Since lifestyle influences High Blood Pressure (HBP), if nothing is done, problem of stroke and sudden deaths would continue. There is need for early diagnosis, prevention and control. Hypertension can be prevented as well as controlled through awareness, modification or elimination of unhealthy lifestyle habits. This informed the decision of the researcher to investigate the knowledge, attitude, lifestyle habits and blood pressure of rural dwellers in Isunjaba of Imo State, through a community intervention programme. Findings would be useful to Community Health Nurses in planning health promotion strategies and policy makers in planning health care programmes in relation to hypertension prevention and control.

\section{Ethical Considerations}

Approval to conduct the study was obtained from the ethical committee, Nnamdi Azikiwe University Teaching Hospital Nnewi (NAUTH/CS/66/VOL.3/009), Anambra State in line with its ethical protocol. Approval to conduct the study was also obtained from the Kings (Ezes') of respective communities and the Chairman of the Local Government Area. Participants were given essential information about the study procedure, duration, its purpose and benefits. Confidentiality of the respondents was assured by not writing names or addresses on the questionnaire and record cards. The right and integrity of the study participants was fully protected and written consent also obtained from each and every one of them. Only those who were willing to participate were included in the study.

\section{Methods and Materials}

The study adopted a quasi-experimental design. Multistage cluster sampling technique was used to select two communities in Isunjaba, assigned into Experimental (EG) and Control groups (CG) by balloting.

*Corresponding author: Osuala Eunice O, Department of Nursing Science, Nnamdi Azikiwe University, Nnewi Campus, South East, Nigeria, Tel: +2348061146538; E-mail: euniceosuala@yahoo.com

Received January 17, 2017; Accepted February 15, 2017; Published February 17, 2017

Citation: Osuala Eunice O (2017) Hypertension Prevention and Control: Effects of a Community Health Nurse-led Intervention. J Health Educ Res Dev 5: 210. doi: 10.4172/2380-5439.1000210

Copyright: ( 2017 Osuala Eunice O. This is an open-access article distributed under the terms of the Creative Commons Attribution License, which permits unrestricted use, distribution, and reproduction in any medium, provided the original author and source are credited. 
A total of 442 rural dwellers (199 from EG and 243 from CG), between ages 20 and 75 years that consented, were selected.

\section{Sample size determination}

This was based on the formula for two proportions [14].

$$
n=\frac{[Z \alpha \sqrt{2 p c(1-p c)}-Z \beta \sqrt{p t(1-p t)+p c(1-p c)}]^{2}}{(p t-p c)^{2}}
$$

\section{Sampling procedure}

Multistage cluster sampling: Multistage sampling technique was adopted: Through purposive sampling, out of the 21 Local Government Areas (LGA) in Imo State, Isu LGA and Isunjaba, one of the five towns in Isu LGA were selected. Using simple random sampling method the required number of communities, clusters of villages, households and participants were selected.

Selection of town: Isu LGA comprises of five towns, Isunjaba (a rural community) one of the towns was chosen purposively for the study based on researcher's observation and familiarity with health issues in the community.

Selection of communities: From the four autonomous communities, using simple random (balloting) technique, two communities were selected. One of the communities was assigned head and the other tail by balloting. With tossing of the coin, the first appearance which was the coat of arms (head) was assigned the experimental group, and Isuobishi (subjects) from this process was assigned the Experimental group (Group A) while Isuokporo (control) was assigned the Control group (Group B). The subjects in Isuobishi were therefore assigned into Group $\mathrm{A}$, and those in Isuokporo into Group B, as experimental and control respectively.

Selection of villages: The eight villages in groups A - Isuobishi were categorised chronologically into two clusters, senior and junior groups. Similarly, same was done for the eleven villages in Group B Isuokporo. Two villages were then randomly selected from each cluster.

Household selection: In each of the two selected villages in the clusters for the study, households were selected, using odd numbers which was determined by tossing the coin. Head (side with coat of arms) was for even while tail (side with unit of amount) was for odd. Households identified as odd from this exercise were selected until required number was got.

Selection of participants: A total of 442 subjects were selected for the study as this figure is the highest calculated sample size figure and as such accommodates the other two values. Experimental group constituted a sample of 199 subjects (45\%) while control constituted 243 (55\%), respectively. This was based on proportion of $968: 1,184$ which makes a total for a study population of 2,152 . In each village, the centre was identified. Tossing the coin, based on the side of the coin, (the head is right and the tail is left) the first house on the street was identified, followed by selection of men and women alternatively from the selected households until required number was got.

A validated structured questionnaire with Correlation coefficient value of 0.76 was used to assess level of knowledge of risk factors of hypertension, attitude to its avoidance and lifestyle in relation to regular BP check, exercise and healthy diet. Maximum scores obtainable for knowledge, attitude and lifestyle were 25,80 and 50 while minimum scores were 0,20 and 0 respectively. Data were collected at baseline, Post Intervention 1 (P1) and post intervention 2 (P2) at three monthly intervals. The Community Health Nursing Intervention (CHNI) consisted of instruction on risk factors for BP, consumption of healthy diet, regular Blood Pressure check and exercise. The CHNI was administered to Experimental Group (EG) for two weeks and none for the Control Group (CG). Descriptive statistics, student $t$ test and paired $\mathrm{t}$ test were used for data analysis with significant level set at $\mathrm{p}=0.05$.

\section{Results}

\section{Socio-demographic characteristics}

Demographic variable showed that ages of respondents ranged from $20-75$ with mean $49.49+14.45$. The age of participants in EG was $49.3+14.5$ years while CG was $50.5+14.4$ years. Ages $50-59$ were greatest in number 100 (22.6\%) followed by ages 60-69 [94 (21.3\%)] while $<30$ were the least with $42(9.5 \%)$ in number. Participants with primary school education had the highest percentage of 38.5. Participants of low income class were $243(57.6 \%)$ while upper class was $28(6.6 \%)$. This is based on house hold income/ day of Lower class $<\mathrm{N} 500(<\$ 3)$, Middle class N 500-2500 (\$3-15), and Upper class $>$ N 2500 (>\$15) (United States Census Bureau household income [15] (Table 1). There were more women in the study than men which show cased a typical rural community in Nigeria.

\section{Objective 1}

To assess participants' knowledge of risk factors, symptoms, management, prevention and complications of hypertension.

Participants' knowledge of risk factors, symptoms, management, prevention and complications of hypertension: Table 2 shows knowledge scores of respondents. At baseline in the experimental group, 74 (73.3\%) knew that blood pressure of 140/90 $\mathrm{mm} \mathrm{Hg}$ is termed Hypertension, while it was $144(87.8 \%)$ at midterm and $137(78.7 \%)$ at the end-line. For Control group it was 96 (64.4\%), 25 (20.3\%) and 24 (14.1\%) respectively. Response on knowledge of alcohol as a risk factor was $80(65.0 \%)$ and $199(100 \%)$ at end-line in experimental while it was $107(64.8 \%)$ and $85(48.3 \%)$ in the control respectively. In the experimental group $108(77.1 \%)$ responded that heat sensation in the head and severe headache is a symptom of hypertension and this improved to 199 (100\%) at end-line. Response by experimental group that regular exercise will help to prevent hypertension was 146 $(86.9 \%)$ and $199(100.0 \%)$ at baseline and end-line respectively while in the control it was $154(74.4 \%)$ and 159 (78.7\%). More participants acknowledged stroke as a complication at end-line. Knowledge of stroke as a complication of hypertension rose from 149 (92.5\%) at baseline to $196(98.5 \%)$ at end-line in the experimental and from 154 $(88.0 \%)$ to $124(85.5 \%)$ in control group.

Respondents' level of knowledge in experimental and Control groups, pre-and post-intervention: In the experimental group $55(27.6 \%)$ and $94(47.2 \%)$ had high and low knowledge at baseline respectively, while it was $131(65.8 \%)$ and none at end-line. In control $61(25.1 \%)$ and $125(51.4 \%)$ had high and low knowledge at baseline respectively while it was $215(88.8 \%)$ at end-line (Table 3). There was increase in level of knowledge in the experimental group at midterm and end-line. High knowledge rose from 55 (27.6\%) to $169(84.9 \%)$ and dropped to $131(65.8 \%)$ respectively. In the control, high knowledge decreased from $61(25.1 \%)$ to $1(0.4 \%)$ and zero respectively (Figure 1$)$. There was significant difference in knowledge of the two groups after intervention, $\mathrm{P}$ value 0.000 but not at baseline $\mathrm{P}$ value 0.68 .

\section{Objective 2}

To assess the attitude of the participants to hypertension and its preventive measures. 
Citation: Osuala Eunice O (2017) Hypertension Prevention and Control: Effects of a Community Health Nurse-led Intervention. J Health Educ Res Dev 5: 210. doi: 10.4172/2380-5439.1000210

Page 3 of 8

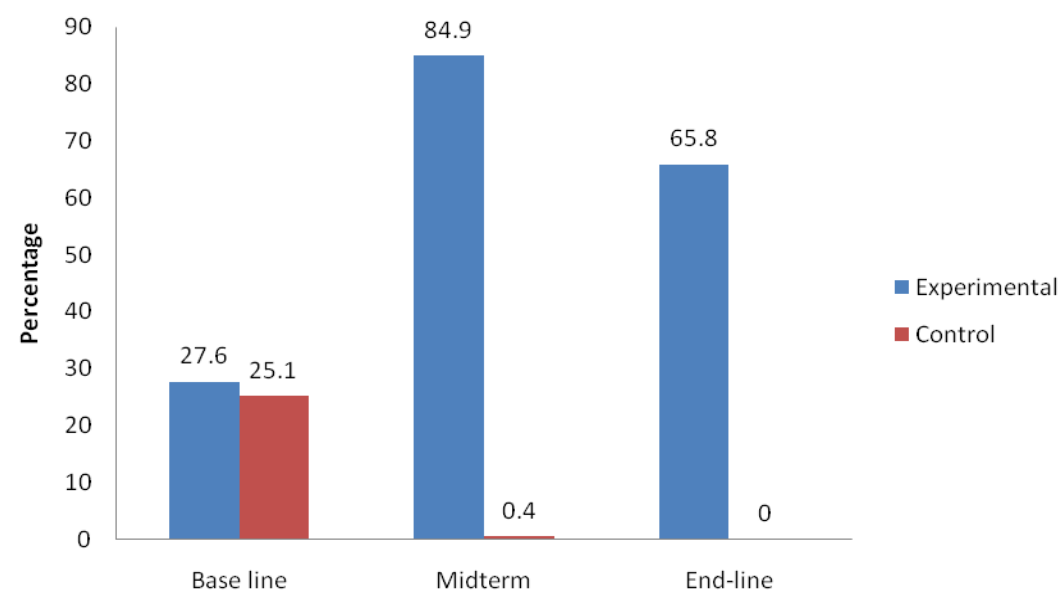

Figure 1: Respondents level of high knowledge, pre and post intervention.

\begin{tabular}{|c|c|c|c|c|c|}
\hline Variables & $\begin{array}{l}\text { Experimental } \\
\quad \mathrm{N}=199\end{array}$ & $\begin{array}{l}\text { Control } \\
\mathrm{N}=243\end{array}$ & $\begin{array}{l}\text { Total } \\
\mathrm{N}=442\end{array}$ & $\mathbf{X}^{2}$ & $P$ value \\
\hline \multicolumn{6}{|c|}{ Sex } \\
\hline Male & $74(37.2)$ & $108(44.4)$ & $182(41.2)$ & 0.123 & 2.380 \\
\hline Female & $125(62.8)$ & $135(55.6)$ & $260(58.8)$ & & \\
\hline Marital Status & & & & 0.365 & 0.947 \\
\hline Married & $166(83.4)$ & $198(81.5)$ & $364(82.4)$ & & \\
\hline Single & $22(11.1)$ & $31(12.8)$ & $53(12.0)$ & & \\
\hline Separated & $2(1.0)$ & $3(1.2)$ & $5(1.1)$ & & \\
\hline Widowed & $9(4.5)$ & $11(4.5)$ & $20(4.5)$ & & \\
\hline Age (Yrs) & & & & 1.280 & 0.937 \\
\hline$<30$ & $21(10.6)$ & $21(8.6)$ & $42(9.5)$ & & \\
\hline $30-39$ & $30(15.1)$ & $37(15.2)$ & $67(15.2)$ & & \\
\hline $40-49$ & $43(21.6)$ & $47(19.3)$ & $90(20.4)$ & & \\
\hline $50-59$ & $45(22.6)$ & $55(22.6)$ & $100(22.6)$ & & \\
\hline $60-69$ & $40(20.1)$ & $54(22.2)$ & $94(21.3)$ & & \\
\hline$>70$ & $20(10.1)$ & $29(11.9)$ & $49(11.1)$ & & \\
\hline Occupation & & & & 9.197 & 0.056 \\
\hline Jobless & $21(10.6)$ & $28(11.5)$ & $49(11.1)$ & & \\
\hline Civil Servant & $45(22.6)$ & $39(16.0)$ & $84(19.0)$ & & \\
\hline Farming & $68(34.2)$ & $114(46.9)$ & $182(41.2)$ & & \\
\hline Petty trading & $37(18.6)$ & $32(13.2)$ & $69(15.6)$ & & \\
\hline Artisan & $28(14.1)$ & $30(12.3)$ & $58(13.1)$ & & \\
\hline Income & & & & 13.988 & 0.003 \\
\hline Upper class & $14(7.0)$ & $14(6.3)$ & $28(6.6)$ & & \\
\hline Middle class & $31(15.6)$ & $11(26.2)$ & $42(10.0)$ & & \\
\hline Lower middle class & $50(25.1)$ & $59(26.5)$ & $109(25.8)$ & & \\
\hline Lower class & $104(52.3)$ & $139(62.3)$ & $243(57.6)$ & & \\
\hline Religion & & & & 7.077 & 0.132 \\
\hline Anglican & $11(5.5)$ & $9(3.7)$ & $20(4.5)$ & & \\
\hline Catholic & 147 (73.9) & $200(82.6)$ & 347 (78.7) & & \\
\hline Pentecostal & $33(16.6)$ & $26(10.7)$ & $59(13.4)$ & & \\
\hline Moslem & $2(1.0)$ & $0(0.0)$ & $2(0.5)$ & & \\
\hline African Traditional & $6(3.0)$ & $7(2.9)$ & $13(2.9)$ & & \\
\hline Level of education & & & & 1.655 & 0.647 \\
\hline Non formal & $29(14.6)$ & $42(17.3)$ & $71(16.1)$ & & \\
\hline Primary & $73(36.7)$ & $97(39.9)$ & $170(38.5)$ & & \\
\hline Secondary & $47(23.6)$ & $51(21.0)$ & $98(22.2)$ & & \\
\hline Tertiary & $50(25.1)$ & $53(21.8)$ & $103(23.3)$ & & \\
\hline
\end{tabular}

Table 1: Socio demographic characteristics of respondents in Experimental and Control at baseline. 
Citation: Osuala Eunice O (2017) Hypertension Prevention and Control: Effects of a Community Health Nurse-led Intervention. J Health Educ Res Dev 5: 210. doi: 10.4172/2380-5439.1000210

Page 4 of 8

\begin{tabular}{|c|c|c|c|c|c|c|c|c|c|}
\hline \multirow[b]{2}{*}{ Statement } & \multicolumn{3}{|c|}{ Baseline } & \multicolumn{3}{|c|}{ Midterm } & \multicolumn{3}{|c|}{ End-line } \\
\hline & $\begin{array}{c}\operatorname{Exp} \\
\text { Freq }(\%)\end{array}$ & $\begin{array}{c}\text { Control Freq } \\
(\%)\end{array}$ & Total & $\begin{array}{c}\text { Exp } \\
\text { Freq (\%) }\end{array}$ & $\begin{array}{l}\text { Control } \\
\text { Freq (\%) }\end{array}$ & Total & $\begin{array}{l}\text { Exp } \\
\text { Freq } \\
(\%)\end{array}$ & $\begin{array}{l}\text { Control } \\
\text { Freq } \\
(\%)\end{array}$ & Total \\
\hline $\begin{array}{c}\text { Definition } \\
\text { Blood pressure of } 140 / 90 \mathrm{mmHg} \text { is } \\
\text { termed hypertension }\end{array}$ & $\begin{array}{c}74 \\
(73.3)\end{array}$ & $\begin{array}{c}96 \\
(64.4)\end{array}$ & $\begin{array}{c}170 \\
(68.0)\end{array}$ & $\begin{array}{c}144 \\
(87.8)\end{array}$ & $\begin{array}{c}25 \\
(20.3)\end{array}$ & $\begin{array}{c}169 \\
(58.9)\end{array}$ & $\begin{array}{c}137 \\
(78.7)\end{array}$ & $\begin{array}{c}24 \\
(14.1)\end{array}$ & $\begin{array}{c}161 \\
(46.8)\end{array}$ \\
\hline $\begin{array}{c}\text { Risk factors } \\
\text { Obesity can bring about } \\
\text { hypertension }\end{array}$ & $\begin{array}{c}91 \\
(70.0)\end{array}$ & $\begin{array}{c}106 \\
(70.0)\end{array}$ & $\begin{array}{c}197 \\
(70.4)\end{array}$ & $\begin{array}{c}196 \\
(98.5)\end{array}$ & $\begin{array}{c}112 \\
(67.1)\end{array}$ & $\begin{array}{c}308 \\
(84.2)\end{array}$ & $\begin{array}{c}199 \\
(100.0)\end{array}$ & $\begin{array}{c}103 \\
(53.6)\end{array}$ & $\begin{array}{c}302 \\
(77.2)\end{array}$ \\
\hline $\begin{array}{l}\text { Tobacco products/snuff can bring } \\
\text { about hypertension }\end{array}$ & $\begin{array}{c}72 \\
(62.1)\end{array}$ & $\begin{array}{c}75 \\
(51.4)\end{array}$ & $\begin{array}{c}147 \\
(56.1)\end{array}$ & $\begin{array}{c}197 \\
(99.0)\end{array}$ & $\begin{array}{c}29 \\
(22.1)\end{array}$ & $\begin{array}{c}226 \\
(68.5)\end{array}$ & $\begin{array}{c}199 \\
(100.0)\end{array}$ & $\begin{array}{c}16 \\
(7.2)\end{array}$ & $\begin{array}{c}215 \\
(50.9)\end{array}$ \\
\hline $\begin{array}{l}\text { Alcohol can bring about } \\
\text { hypertension }\end{array}$ & $\begin{array}{c}80 \\
(65.0)\end{array}$ & $\begin{array}{c}107 \\
(64.8)\end{array}$ & $\begin{array}{c}187 \\
(64.9)\end{array}$ & $\begin{array}{c}199 \\
(100.0)\end{array}$ & $\begin{array}{c}87 \\
(59.6)\end{array}$ & $\begin{array}{c}286 \\
(82.9)\end{array}$ & $\begin{array}{c}199 \\
(100.0)\end{array}$ & $\begin{array}{c}85 \\
(48.3)\end{array}$ & $\begin{array}{c}284 \\
(75.7)\end{array}$ \\
\hline $\begin{array}{l}\text { Use of table salt can bring about } \\
\text { hypertension }\end{array}$ & $\begin{array}{c}87 \\
(65.9)\end{array}$ & $\begin{array}{c}89 \\
(57.1)\end{array}$ & $\begin{array}{c}176 \\
(61.1)\end{array}$ & $\begin{array}{c}195 \\
(98.0)\end{array}$ & $\begin{array}{c}45 \\
(22.8)\end{array}$ & $\begin{array}{c}240 \\
(60.6)\end{array}$ & $\begin{array}{c}199 \\
(100.0)\end{array}$ & $\begin{array}{c}38 \\
(16.1)\end{array}$ & $\begin{array}{c}237 \\
(54.5)\end{array}$ \\
\hline Hypertension is hereditary & $\begin{array}{c}64 \\
(50.0)\end{array}$ & $\begin{array}{c}85 \\
(55.2)\end{array}$ & $\begin{array}{c}149 \\
(52.8)\end{array}$ & $\begin{array}{c}165 \\
(87.3)\end{array}$ & $\begin{array}{c}50 \\
(32.9)\end{array}$ & $\begin{array}{c}215 \\
(63.0)\end{array}$ & $\begin{array}{c}196 \\
(99.5)\end{array}$ & $\begin{array}{c}43 \\
(27.4)\end{array}$ & $\begin{array}{c}239 \\
(67.5)\end{array}$ \\
\hline $\begin{array}{l}\text { Signs and symptoms } \\
\text { Heat sensation in the head with } \\
\text { severe headache is a symptom of } \\
\text { hypertension }\end{array}$ & $\begin{array}{c}108 \\
(77.1)\end{array}$ & $\begin{array}{c}156 \\
(85.7)\end{array}$ & $\begin{array}{c}264 \\
(82.0)\end{array}$ & $\begin{array}{c}198 \\
(64.1)\end{array}$ & $\begin{array}{c}111 \\
(72.5)\end{array}$ & $\begin{array}{c}309 \\
(88.0)\end{array}$ & $\begin{array}{c}199 \\
(100.0)\end{array}$ & $\begin{array}{c}107 \\
(54.6)\end{array}$ & $\begin{array}{c}306 \\
(77.5)\end{array}$ \\
\hline $\begin{array}{l}\text { Hypertension may come with pain } \\
\text { side of neck }\end{array}$ & $\begin{array}{c}73 \\
(64.0)\end{array}$ & $\begin{array}{c}102 \\
(70.8)\end{array}$ & $\begin{array}{c}175 \\
(67.8)\end{array}$ & $\begin{array}{c}192 \\
(98.0)\end{array}$ & $\begin{array}{c}28 \\
(23.3)\end{array}$ & $\begin{array}{c}220 \\
(69.6)\end{array}$ & $\begin{array}{c}199 \\
(100.0)\end{array}$ & $\begin{array}{c}21 \\
(10.5)\end{array}$ & $\begin{array}{c}220 \\
(55.1)\end{array}$ \\
\hline $\begin{array}{c}\text { Prevention } \\
\text { Regular exercise prevents } \\
\text { Hypertension }\end{array}$ & $\begin{array}{c}146 \\
(86.9)\end{array}$ & $\begin{array}{c}154 \\
(74.4)\end{array}$ & $\begin{array}{c}300 \\
(80.0)\end{array}$ & $\begin{array}{c}196 \\
(98.5)\end{array}$ & $\begin{array}{c}168 \\
(82.4)\end{array}$ & $\begin{array}{c}364 \\
(90.3)\end{array}$ & $\begin{array}{c}199 \\
(100.0)\end{array}$ & $\begin{array}{c}159 \\
(78.7)\end{array}$ & $\begin{array}{c}358 \\
(89.3)\end{array}$ \\
\hline $\begin{array}{c}\text { Management } \\
\text { Hypertension is best managed in } \\
\text { hospital }\end{array}$ & $\begin{array}{c}168 \\
(88.9)\end{array}$ & $\begin{array}{c}207 \\
(89.2)\end{array}$ & $\begin{array}{c}375 \\
(89.1)\end{array}$ & $\begin{array}{c}194 \\
(97.5)\end{array}$ & $\begin{array}{c}214 \\
(91.1)\end{array}$ & $\begin{array}{c}408 \\
(94.0)\end{array}$ & $\begin{array}{c}197 \\
(99.5)\end{array}$ & $\begin{array}{c}209 \\
(88.9)\end{array}$ & $\begin{array}{c}406 \\
(93.8)\end{array}$ \\
\hline $\begin{array}{c}\text { Complications } \\
\text { Hypertension can cause eye } \\
\text { damage }\end{array}$ & $\begin{array}{c}121 \\
(81.2)\end{array}$ & $\begin{array}{c}146 \\
(80.2)\end{array}$ & $\begin{array}{c}267 \\
(80.7)\end{array}$ & $\begin{array}{c}197 \\
(72.4)\end{array}$ & $\begin{array}{c}75 \\
(51.7)\end{array}$ & $\begin{array}{c}272 \\
(79.1)\end{array}$ & $\begin{array}{c}198 \\
(99.5)\end{array}$ & $\begin{array}{c}51 \\
(23.5)\end{array}$ & $\begin{array}{c}249 \\
(59.9)\end{array}$ \\
\hline $\begin{array}{l}\text { Stroke is complication of } \\
\text { hypertension }\end{array}$ & $\begin{array}{c}149 \\
(92.5)\end{array}$ & $\begin{array}{c}154 \\
(88.0)\end{array}$ & $\begin{array}{c}303 \\
(90.2)\end{array}$ & $\begin{array}{c}197 \\
(99.5)\end{array}$ & $\begin{array}{c}124 \\
(85.5)\end{array}$ & $\begin{array}{c}321 \\
(93.6)\end{array}$ & $\begin{array}{c}196 \\
(98.5)\end{array}$ & $\begin{array}{c}126 \\
(74.1)\end{array}$ & $\begin{array}{c}322 \\
(87.3)\end{array}$ \\
\hline
\end{tabular}

Table 2: Participants' knowledge of risk factors, symptoms, management, prevention and complications of hypertension.

\begin{tabular}{|c|c|c|c|c|c|c|c|c|c|c|c|}
\hline \multirow{2}{*}{ Statement } & \multicolumn{6}{|c|}{ Experimental } & \multicolumn{5}{|c|}{ Control } \\
\hline & & SA & A & $\mathrm{D}$ & SD & Total & SA & A & $\mathrm{D}$ & SD & Total \\
\hline \multirow{3}{*}{ HBP is a serious ailment } & $1^{\text {st }}$ & $\begin{array}{c}144 \\
72.4 \%\end{array}$ & $\begin{array}{c}45 \\
22.6 \%\end{array}$ & $\begin{array}{c}9 \\
4.5 \%\end{array}$ & $\begin{array}{c}1 \\
0.5 \%\end{array}$ & $\begin{array}{c}199 \\
100.0\end{array}$ & $\begin{array}{c}172 \\
70.8 \%\end{array}$ & $\begin{array}{c}55 \\
22.6 \%\end{array}$ & $\begin{array}{c}12 \\
4.9 \%\end{array}$ & $\begin{array}{c}4 \\
1.6 \%\end{array}$ & $\begin{array}{c}243 \\
100.0\end{array}$ \\
\hline & $2^{\text {nd }}$ & $\begin{array}{l}195 \\
98.0\end{array}$ & $\begin{array}{c}4 \\
2.0\end{array}$ & $\begin{array}{c}0 \\
0.0\end{array}$ & $\begin{array}{l}0.0 \\
0.0\end{array}$ & $\begin{array}{c}199 \\
100.0\end{array}$ & $\begin{array}{r}196 \\
81.0\end{array}$ & $\begin{array}{c}38 \\
15.7\end{array}$ & $\begin{array}{c}2 \\
0.8\end{array}$ & $\begin{array}{c}6 \\
2.5\end{array}$ & $\begin{array}{c}242 \\
100.0\end{array}$ \\
\hline & $3^{\text {rd }}$ & $\begin{array}{l}196 \\
99.0\end{array}$ & $\begin{array}{c}2 \\
1.0\end{array}$ & $\begin{array}{c}0 \\
0.0\end{array}$ & $\begin{array}{l}0.0 \\
0.0\end{array}$ & $\begin{array}{c}198 \\
100.0\end{array}$ & $\begin{array}{r}195 \\
80.6\end{array}$ & $\begin{array}{c}39 \\
16.1\end{array}$ & $\begin{array}{c}2 \\
0.8\end{array}$ & $\begin{array}{c}6 \\
2.5\end{array}$ & $\begin{array}{c}242 \\
100.0\end{array}$ \\
\hline \multirow{3}{*}{ You will readily give up a habit that can lead to HBP } & $1^{\text {st }}$ & $\begin{array}{c}99 \\
49.7\end{array}$ & $\begin{array}{c}78 \\
39.2\end{array}$ & $\begin{array}{l}13 \\
6.5\end{array}$ & $\begin{array}{c}9 \\
4.5\end{array}$ & $\begin{array}{c}199 \\
100.0\end{array}$ & $\begin{array}{c}104 \\
42.8\end{array}$ & $\begin{array}{r}110 \\
45.3\end{array}$ & $\begin{array}{l}19 \\
7.8\end{array}$ & $\begin{array}{l}10 \\
4.1\end{array}$ & $\begin{array}{c}243 \\
100.0\end{array}$ \\
\hline & $2^{\text {nd }}$ & $\begin{array}{c}131 \\
65.8\end{array}$ & $\begin{array}{c}65 \\
32.7\end{array}$ & $\begin{array}{c}1 \\
0.5\end{array}$ & $\begin{array}{c}2 \\
1.0\end{array}$ & $\begin{array}{c}199 \\
100.0\end{array}$ & $\begin{array}{r}135 \\
55.8\end{array}$ & $\begin{array}{c}77 \\
31.8\end{array}$ & $\begin{array}{l}16 \\
6.6\end{array}$ & $\begin{array}{c}14 \\
5.8\end{array}$ & 242 \\
\hline & $3^{\text {rd }}$ & $\begin{array}{r}179 \\
89.9\end{array}$ & $\begin{array}{l}18 \\
9.0\end{array}$ & $\begin{array}{c}0 \\
0.0\end{array}$ & $\begin{array}{c}2 \\
1.0\end{array}$ & $\begin{array}{c}199 \\
100.0\end{array}$ & $\begin{array}{c}137 \\
56.8\end{array}$ & $\begin{array}{c}75 \\
31.1\end{array}$ & $\begin{array}{l}15 \\
6.2\end{array}$ & $\begin{array}{c}14 \\
5.8\end{array}$ & 241 \\
\hline \multirow{3}{*}{ Regular BP check, even when well if tool is available } & $1^{\text {st }}$ & $\begin{array}{c}91 \\
45.7\end{array}$ & $\begin{array}{c}73 \\
36.7\end{array}$ & $\begin{array}{l}15 \\
7.5\end{array}$ & $\begin{array}{c}20 \\
10.1\end{array}$ & $\begin{array}{c}199 \\
100.0\end{array}$ & $\begin{array}{c}91 \\
37.4\end{array}$ & $\begin{array}{c}89 \\
36.6\end{array}$ & $\begin{array}{l}15 \\
6.2\end{array}$ & $\begin{array}{c}48 \\
19.8\end{array}$ & $\begin{array}{c}243 \\
100.0\end{array}$ \\
\hline & $2^{\text {nd }}$ & $\begin{array}{c}89 \\
44.7\end{array}$ & $\begin{array}{c}104 \\
52.3\end{array}$ & $\begin{array}{c}3 \\
1.5\end{array}$ & $\begin{array}{c}3 \\
1.5\end{array}$ & $\begin{array}{c}199 \\
100.0\end{array}$ & $\begin{array}{c}49 \\
20.2\end{array}$ & $\begin{array}{c}29 \\
12.0\end{array}$ & $\begin{array}{c}91 \\
37.6\end{array}$ & $\begin{array}{c}73 \\
30.2\end{array}$ & $\begin{array}{c}242 \\
100.0\end{array}$ \\
\hline & $3^{\text {rd }}$ & $\begin{array}{c}176 \\
88.4\end{array}$ & $\begin{array}{l}15 \\
7.5\end{array}$ & $\begin{array}{c}2 \\
1.0\end{array}$ & $\begin{array}{c}6 \\
3.0\end{array}$ & $\begin{array}{c}199 \\
100.0\end{array}$ & $\begin{array}{l}20 \\
8.3\end{array}$ & $\begin{array}{c}50 \\
20.7\end{array}$ & $\begin{array}{c}87 \\
36.0\end{array}$ & $\begin{array}{c}85 \\
35.1\end{array}$ & $\begin{array}{c}242 \\
100.0\end{array}$ \\
\hline \multirow{3}{*}{ Use of table salt is good if food is tasteless } & $1^{\text {st }}$ & $\begin{array}{c}41 \\
20.6\end{array}$ & $\begin{array}{c}57 \\
28.6\end{array}$ & $\begin{array}{c}75 \\
37.7\end{array}$ & $\begin{array}{c}26 \\
13.1\end{array}$ & $\begin{array}{c}199 \\
100.0\end{array}$ & $\begin{array}{c}87 \\
35.8\end{array}$ & $\begin{array}{c}77 \\
31.7\end{array}$ & $\begin{array}{c}53 \\
21.8\end{array}$ & $\begin{array}{c}26 \\
10.7\end{array}$ & $\begin{array}{c}243 \\
100.0\end{array}$ \\
\hline & $2^{\text {nd }}$ & $\begin{array}{c}22 \\
11.1\end{array}$ & $\begin{array}{c}32 \\
16.2 \\
\end{array}$ & $\begin{array}{c}93 \\
47.0\end{array}$ & $\begin{array}{c}51 \\
25.8\end{array}$ & $\begin{array}{c}198 \\
100.0\end{array}$ & $\begin{array}{r}175 \\
72.3 \\
\end{array}$ & $\begin{array}{c}38 \\
15.7\end{array}$ & $\begin{array}{r}10 \\
4.1\end{array}$ & $\begin{array}{r}19 \\
7.9\end{array}$ & $\begin{array}{c}242 \\
100.0\end{array}$ \\
\hline & $3^{\text {rd }}$ & $\begin{array}{c}36 \\
18.2\end{array}$ & $\begin{array}{c}14 \\
7.1\end{array}$ & $\begin{array}{l}12 \\
6.1\end{array}$ & $\begin{array}{r}136 \\
68.7\end{array}$ & $\begin{array}{c}198 \\
100.0\end{array}$ & $\begin{array}{r}193 \\
79.8\end{array}$ & $\begin{array}{l}19 \\
7.9\end{array}$ & $\begin{array}{l}22 \\
9.1\end{array}$ & $\begin{array}{c}8 \\
3.3\end{array}$ & $\begin{array}{c}242 \\
100.0\end{array}$ \\
\hline
\end{tabular}


Citation: Osuala Eunice O (2017) Hypertension Prevention and Control: Effects of a Community Health Nurse-led Intervention. J Health Educ Res Dev 5: 210. doi: 10.4172/2380-5439.1000210

Page 5 of 8

\begin{tabular}{|c|c|c|c|c|c|c|c|c|c|c|c|}
\hline \multirow{3}{*}{ HBP affects women most } & $1^{\text {st }}$ & $\begin{array}{c}33 \\
16.6\end{array}$ & $\begin{array}{c}45 \\
22.6\end{array}$ & $\begin{array}{c}93 \\
46.7\end{array}$ & $\begin{array}{c}28 \\
14.1\end{array}$ & $\begin{array}{c}199 \\
100.0\end{array}$ & $\begin{array}{c}71 \\
29.3\end{array}$ & $\begin{array}{c}42 \\
17.4\end{array}$ & $\begin{array}{c}86 \\
35.5\end{array}$ & $\begin{array}{c}43 \\
17.8\end{array}$ & $\begin{array}{c}242 \\
100.0\end{array}$ \\
\hline & $2^{\text {nd }}$ & $\begin{array}{c}63 \\
31.7\end{array}$ & $\begin{array}{c}23 \\
11.6\end{array}$ & $\begin{array}{c}66 \\
33.2\end{array}$ & $\begin{array}{c}47 \\
23.6\end{array}$ & $\begin{array}{c}199 \\
100.0\end{array}$ & $\begin{array}{c}130 \\
53.7\end{array}$ & $\begin{array}{l}20 \\
8.3\end{array}$ & $\begin{array}{c}32 \\
13.2\end{array}$ & $\begin{array}{c}60 \\
24.8\end{array}$ & $\begin{array}{c}242 \\
100.0\end{array}$ \\
\hline & $3^{\text {rd }}$ & $\begin{array}{c}83 \\
41.9\end{array}$ & $\begin{array}{c}6 \\
3.020\end{array}$ & $\begin{array}{l}18 \\
9.1\end{array}$ & $\begin{array}{c}91 \\
46.0\end{array}$ & $\begin{array}{c}198 \\
100.0\end{array}$ & $\begin{array}{r}146 \\
60.3\end{array}$ & $\begin{array}{c}8 \\
3.3\end{array}$ & $\begin{array}{c}63 \\
26.0\end{array}$ & $\begin{array}{c}25 \\
10.3\end{array}$ & $\begin{array}{c}242 \\
100.0\end{array}$ \\
\hline \multirow{3}{*}{ HBP does not affect youth } & $1^{\text {st }}$ & $\begin{array}{c}32 \\
16.1\end{array}$ & $\begin{array}{c}56 \\
28.1\end{array}$ & $\begin{array}{c}87 \\
43.7\end{array}$ & $\begin{array}{c}24 \\
12.1\end{array}$ & $\begin{array}{c}199 \\
100.0\end{array}$ & $\begin{array}{c}58 \\
24.0\end{array}$ & $\begin{array}{c}52 \\
21.5\end{array}$ & $\begin{array}{c}96 \\
39.7\end{array}$ & $\begin{array}{c}36 \\
14.9\end{array}$ & $\begin{array}{c}242 \\
100.0\end{array}$ \\
\hline & $2^{\text {nd }}$ & $\begin{array}{c}42 \\
21.2\end{array}$ & $\begin{array}{l}12 \\
6.1\end{array}$ & $\begin{array}{c}85 \\
42.9\end{array}$ & $\begin{array}{c}59 \\
29.8\end{array}$ & $\begin{array}{c}198 \\
100.0\end{array}$ & $\begin{array}{c}160 \\
66.1\end{array}$ & $\begin{array}{c}31 \\
12.8\end{array}$ & $\begin{array}{c}14 \\
5.8\end{array}$ & $\begin{array}{c}37 \\
15.3\end{array}$ & $\begin{array}{c}242 \\
100.0\end{array}$ \\
\hline & $3^{\text {rd }}$ & $\begin{array}{c}56 \\
28.3\end{array}$ & $\begin{array}{c}5 \\
2.5\end{array}$ & $\begin{array}{l}13 \\
6.6\end{array}$ & $\begin{array}{c}124 \\
62.6\end{array}$ & $\begin{array}{c}198 \\
100.0\end{array}$ & $\begin{array}{r}183 \\
75.6 \\
\end{array}$ & $\begin{array}{l}17 \\
7.0\end{array}$ & $\begin{array}{c}35 \\
14.5\end{array}$ & $\begin{array}{c}7 \\
2.9\end{array}$ & $\begin{array}{c}242 \\
100.0\end{array}$ \\
\hline \multirow{3}{*}{$\begin{array}{l}\text { HBP will develop whether one adopts a positive health } \\
\qquad \text { action or not }\end{array}$} & $1^{\text {st }}$ & $\begin{array}{c}40 \\
20.1\end{array}$ & $\begin{array}{c}59 \\
29.6\end{array}$ & $\begin{array}{c}67 \\
33.7\end{array}$ & $\begin{array}{c}33 \\
16.6\end{array}$ & $\begin{array}{c}199 \\
100.0\end{array}$ & $\begin{array}{c}91 \\
37.4\end{array}$ & $\begin{array}{c}64 \\
26.3\end{array}$ & $\begin{array}{c}47 \\
19.3\end{array}$ & $\begin{array}{c}41 \\
16.9\end{array}$ & $\begin{array}{c}243 \\
100.0\end{array}$ \\
\hline & $2^{\text {nd }}$ & $\begin{array}{c}31 \\
15.6\end{array}$ & $\begin{array}{c}9 \\
4.5\end{array}$ & $\begin{array}{c}92 \\
46.2\end{array}$ & $\begin{array}{c}67 \\
33.7\end{array}$ & $\begin{array}{c}199 \\
100.0\end{array}$ & $\begin{array}{c}168 \\
69.4\end{array}$ & $\begin{array}{c}43 \\
17.8\end{array}$ & $\begin{array}{c}9 \\
3.7\end{array}$ & $\begin{array}{l}22 \\
9.1\end{array}$ & $\begin{array}{c}242 \\
100.0\end{array}$ \\
\hline & $3^{\text {rd }}$ & $\begin{array}{c}42 \\
21.3\end{array}$ & $\begin{array}{c}3 \\
1.5\end{array}$ & $\begin{array}{l}10 \\
5.1\end{array}$ & $\begin{array}{c}142 \\
72.1\end{array}$ & $\begin{array}{c}197 \\
100.0\end{array}$ & $\begin{array}{l}209 \\
86.4\end{array}$ & $\begin{array}{c}11 \\
4.5\end{array}$ & $\begin{array}{l}16 \\
6.6\end{array}$ & $\begin{array}{c}6 \\
2.5\end{array}$ & $\begin{array}{c}242 \\
100.0\end{array}$ \\
\hline
\end{tabular}

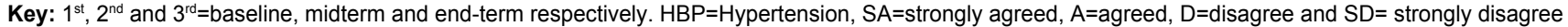

Table 3: Attitude of the participants to hypertension and its preventive measures.

\begin{tabular}{|c|c|c|c|c|c|c|c|c|c|}
\hline \multirow{2}{*}{ Statement } & \multirow[b]{2}{*}{ Phase } & \multicolumn{4}{|c|}{ Experimental } & \multicolumn{4}{|c|}{ Control } \\
\hline & & Always & Occasionally & Never & Total & Always & Occasionally & Never & Total \\
\hline \multirow{3}{*}{ You do regular exercise } & $1^{\text {st }}$ & $\begin{array}{c}89 \\
44.7\end{array}$ & $\begin{array}{c}90 \\
45.2\end{array}$ & $\begin{array}{c}20 \\
10.1\end{array}$ & $\begin{array}{c}199 \\
100.0\end{array}$ & $\begin{array}{r}106 \\
43.6\end{array}$ & $\begin{array}{c}80 \\
32.9\end{array}$ & $\begin{array}{c}57 \\
23.5\end{array}$ & $\begin{array}{c}243 \\
100.0\end{array}$ \\
\hline & $2^{\text {nd }}$ & $\begin{array}{l}185 \\
93.0\end{array}$ & $\begin{array}{c}12 \\
29.3\end{array}$ & $\begin{array}{c}2 \\
1.0\end{array}$ & $\begin{array}{c}199 \\
100.0\end{array}$ & $\begin{array}{c}4 \\
1.7\end{array}$ & $\begin{array}{c}29 \\
12.0\end{array}$ & $\begin{array}{l}209 \\
86.4\end{array}$ & 242 \\
\hline & $3^{\text {rd }}$ & $\begin{array}{l}193 \\
99.0\end{array}$ & $\begin{array}{c}2 \\
1.0\end{array}$ & $\begin{array}{c}0 \\
0.0\end{array}$ & $\begin{array}{c}195 \\
100.0\end{array}$ & $\begin{array}{c}0 \\
0.0\end{array}$ & $\begin{array}{c}19 \\
90.5\end{array}$ & $\begin{array}{l}223 \\
92.1\end{array}$ & $\begin{array}{c}242 \\
100.0\end{array}$ \\
\hline \multirow{3}{*}{$\begin{array}{l}\text { In the last } 3 \text { months you } \\
\text { have checked your BP }\end{array}$} & $1^{\text {st }}$ & $\begin{array}{c}27 \\
13.6\end{array}$ & $\begin{array}{c}67 \\
33.7\end{array}$ & $\begin{array}{l}105 \\
52.8\end{array}$ & $\begin{array}{c}199 \\
100.0\end{array}$ & $\begin{array}{c}37 \\
15.2\end{array}$ & $\begin{array}{c}62 \\
25.5\end{array}$ & $\begin{array}{r}144 \\
59.3\end{array}$ & $\begin{array}{c}243 \\
100.0\end{array}$ \\
\hline & $2^{\text {nd }}$ & $\begin{array}{l}192 \\
96.5\end{array}$ & $\begin{array}{c}6 \\
3.0\end{array}$ & $\begin{array}{c}1 \\
0.5\end{array}$ & $\begin{array}{c}199 \\
100.0\end{array}$ & $\begin{array}{c}72 \\
29.8\end{array}$ & $\begin{array}{c}4 \\
1.7\end{array}$ & $\begin{array}{l}166 \\
68.6\end{array}$ & $\begin{array}{c}242 \\
100.0\end{array}$ \\
\hline & $3^{\text {rd }}$ & $\begin{array}{l}115 \\
59.0\end{array}$ & $\begin{array}{c}78 \\
40.0\end{array}$ & $\begin{array}{c}2 \\
1.0\end{array}$ & $\begin{array}{c}195 \\
100.0\end{array}$ & $\begin{array}{c}1 \\
0.4\end{array}$ & $\begin{array}{l}17 \\
7.0\end{array}$ & $\begin{array}{l}224 \\
92.6\end{array}$ & $\begin{array}{c}242 \\
100.0\end{array}$ \\
\hline \multirow{3}{*}{$\begin{array}{l}\text { You have time for rest } \\
\text { during the day }\end{array}$} & $1^{\text {st }}$ & $\begin{array}{c}23 \\
11.6\end{array}$ & $\begin{array}{l}126 \\
63.3\end{array}$ & $\begin{array}{c}50 \\
25.1\end{array}$ & $\begin{array}{c}199 \\
100.0\end{array}$ & $\begin{array}{c}31 \\
12.8\end{array}$ & $\begin{array}{l}148 \\
60.9\end{array}$ & $\begin{array}{c}64 \\
26.3\end{array}$ & $\begin{array}{c}243 \\
100.0\end{array}$ \\
\hline & $2^{\text {nd }}$ & $\begin{array}{c}39 \\
19.6\end{array}$ & $\begin{array}{l}150 \\
75.4\end{array}$ & $\begin{array}{l}10 \\
5.0\end{array}$ & $\begin{array}{c}199 \\
100.0\end{array}$ & $\begin{array}{c}7 \\
2.9\end{array}$ & $\begin{array}{c}81 \\
33.5\end{array}$ & $\begin{array}{c}154 \\
63.6\end{array}$ & $\begin{array}{c}242 \\
100.0\end{array}$ \\
\hline & $3^{\text {rd }}$ & $\begin{array}{l}115 \\
59.0\end{array}$ & $\begin{array}{c}78 \\
40.0\end{array}$ & $\begin{array}{c}2 \\
1.0\end{array}$ & $\begin{array}{c}195 \\
100.0\end{array}$ & $\begin{array}{c}1 \\
0.4\end{array}$ & $\begin{array}{l}17 \\
7.0\end{array}$ & $\begin{array}{l}224 \\
92.6\end{array}$ & $\begin{array}{c}242 \\
100.0\end{array}$ \\
\hline \multirow{3}{*}{ You take fruits every day } & $1^{\text {st }}$ & $\begin{array}{c}65 \\
32.7\end{array}$ & $\begin{array}{r}130 \\
65.3\end{array}$ & $\begin{array}{c}4 \\
2.0\end{array}$ & $\begin{array}{c}199 \\
100.0\end{array}$ & $\begin{array}{c}63 \\
25.9\end{array}$ & $\begin{array}{r}175 \\
72.0\end{array}$ & $\begin{array}{c}5 \\
2.1\end{array}$ & $\begin{array}{c}243 \\
100.0\end{array}$ \\
\hline & $2^{\text {nd }}$ & $\begin{array}{l}184 \\
92.5\end{array}$ & $\begin{array}{l}13 \\
6.5\end{array}$ & $\begin{array}{c}2 \\
1.0\end{array}$ & $\begin{array}{c}199 \\
100.0\end{array}$ & $\begin{array}{c}2 \\
0.8\end{array}$ & $\begin{array}{l}234 \\
96.7\end{array}$ & $\begin{array}{c}6 \\
2.5\end{array}$ & $\begin{array}{c}8 \\
1.8\end{array}$ \\
\hline & $3^{\text {rd }}$ & $\begin{array}{l}193 \\
99.0\end{array}$ & $\begin{array}{c}0 \\
0.0\end{array}$ & $\begin{array}{c}2 \\
1.0\end{array}$ & $\begin{array}{c}195 \\
100.0\end{array}$ & $\begin{array}{c}0 \\
0.0\end{array}$ & $\begin{array}{l}232 \\
95.9\end{array}$ & $\begin{array}{l}10 \\
4.1\end{array}$ & $\begin{array}{c}242 \\
100.0\end{array}$ \\
\hline \multirow{3}{*}{ You add salt at table } & $1^{\text {st }}$ & $\begin{array}{c}24 \\
12.1\end{array}$ & $\begin{array}{c}74 \\
37.2\end{array}$ & $\begin{array}{c}101 \\
50.0\end{array}$ & $\begin{array}{c}199 \\
100.0\end{array}$ & $\begin{array}{c}33 \\
13.6\end{array}$ & $\begin{array}{r}118 \\
48.6\end{array}$ & $\begin{array}{c}92 \\
37.9\end{array}$ & $\begin{array}{c}243 \\
100.0\end{array}$ \\
\hline & $2^{\text {nd }}$ & $\begin{array}{c}2 \\
1.0\end{array}$ & $\begin{array}{c}7 \\
3.5\end{array}$ & $\begin{array}{r}190 \\
95.5\end{array}$ & $\begin{array}{c}199 \\
100.0\end{array}$ & $\begin{array}{c}32 \\
13.3\end{array}$ & $\begin{array}{l}154 \\
63.9\end{array}$ & $\begin{array}{c}55 \\
22.8\end{array}$ & $\begin{array}{c}241 \\
100.0\end{array}$ \\
\hline & $3^{\text {rd }}$ & $\begin{array}{c}2 \\
1.0\end{array}$ & $\begin{array}{c}0 \\
0.0\end{array}$ & $\begin{array}{l}192 \\
99.0\end{array}$ & $\begin{array}{c}194 \\
100.0\end{array}$ & $\begin{array}{c}32 \\
13.2\end{array}$ & $\begin{array}{c}171 \\
70.7\end{array}$ & $\begin{array}{c}39 \\
16.1\end{array}$ & $\begin{array}{c}242 \\
100.0\end{array}$ \\
\hline \multirow{3}{*}{$\begin{array}{l}\text { You consider your work } \\
\text { stressful }\end{array}$} & $1^{\text {st }}$ & $\begin{array}{c}24 \\
12.1\end{array}$ & $\begin{array}{c}84 \\
42.2 \\
\end{array}$ & $\begin{array}{c}91 \\
45.7\end{array}$ & $\begin{array}{c}199 \\
100.0\end{array}$ & $\begin{array}{c}63 \\
25.9\end{array}$ & $\begin{array}{r}101 \\
41.6\end{array}$ & $\begin{array}{c}79 \\
32.5\end{array}$ & $\begin{array}{c}243 \\
100.0\end{array}$ \\
\hline & $2^{\text {nd }}$ & $\begin{array}{c}6 \\
3.0\end{array}$ & $\begin{array}{c}93 \\
46.7\end{array}$ & $\begin{array}{r}100 \\
50.3\end{array}$ & $\begin{array}{c}199 \\
100.0\end{array}$ & $\begin{array}{c}51 \\
21.1\end{array}$ & $\begin{array}{r}106 \\
43.8\end{array}$ & $\begin{array}{c}85 \\
35.1\end{array}$ & $\begin{array}{c}242 \\
100.0\end{array}$ \\
\hline & $3^{\text {rd }}$ & $\begin{array}{c}73 \\
37.4\end{array}$ & $\begin{array}{c}61 \\
31.3\end{array}$ & $\begin{array}{c}61 \\
31.3\end{array}$ & $\begin{array}{c}195 \\
100.0\end{array}$ & $\begin{array}{c}87 \\
36.0\end{array}$ & $\begin{array}{c}92 \\
38.0\end{array}$ & $\begin{array}{c}63 \\
26.0\end{array}$ & $\begin{array}{c}242 \\
100.0\end{array}$ \\
\hline
\end{tabular}

Table 4: Participants' lifestyle habits in relation to hypertension.

Attitude of the participants to hypertension and its preventive measures: At baseline 206 (46.6\%) of the respondents had positive attitude score while $236(53.4 \%)$ had negative. Out of the 442 participants, $316(71.5 \%)$ agreed that hypertension is a serious ailment. Two hundred and three (45.9\%) will give up habit if related to hypertension. $182(41.2 \%)$ will have regular blood pressure checks even when well if measuring tool is available while for $90(20.4 \%)$ stated that hypertension is not for young people. One hundred and twenty-eight (29.0\%) stated that table salt should be used if food is tasteless. $131(29.6 \%)$ also strongly believed that one will develop hypertension, adoption of healthy lifestyle or not. Attitude improved in the experimental group as positive attitude score rose from 119 
(59.8\%) at baseline to $185(90.3 \%)$ and $173(86.9 \%)$ at midterm and end-line respectively while in the control the score decreased from 117 $(48.1 \%)$ at baseline to $40(16.5 \%)$ and $49(20.2 \%)$ at midterm and endline respectively (Table 3$)$.

Respondents' attitude scores to hypertension in experimental and control, pre and post intervention: In the experimental group, 119 (59.8\%), $185(93.0 \%)$ and $173(86.9 \%)$ and of the respondents had positive attitude score while in the control it was 117 (48.1\%), 40 (16.5\%) and $49(20.2 \%)$ at baseline, midterm and end-line respectively (Table 4) 80 (40.2\%), 14 (7.0\%) and 26 (13.1\%) of the respondents had negative attitude score in experimental group while in the control it was $126(51.9 \%), 202(83.5 \%)$ and $193(79.8 \%)$ at baseline, midterm and end-line respectively.

\section{Objective 3}

To identify participants' lifestyles habits in relation to hypertension.

Participants' lifestyles habits in relation to hypertension: Identified lifestyle habits among respondents include use of table salt, alcohol, kola, and cigarette and snuff consumption. Among the experimental group, regular exercise was done by $89(44.7 \%)$ at baseline and $193(99.0 \%)$ at end-line while in control it was 106 $(43.6 \%)$ and $0(0 \%)$ at baseline and end-line respectively. Within three months' participants in the experimental group that checked their blood pressure were $27(13.6 \%)$ and $195(100.0 \%)$ at baseline and end-line respectively while among control it was $37(15.2 \%)$ and 23 (9.5\%) respectively. At baseline fruit was never taken by 4 (2.0\%) and 5 (2.1\%) in the experimental and control respectively and even after the intervention. Few of the respondents in both experimental $24(12.1 \%)$ and control 33 (13.6\%) groups use salt at table. The number dropped in the experimental to $2(1.0 \%)$ at end-line of intervention (Table 4).

Respondents' lifestyle scores to hypertension in experimental and control, pre and post intervention: Respondents with positive lifestyle habits score, rose from 119 (59.8\%) at baseline 195 (98.0\%) to $195(100 \%)$ at end-line in the experimental group. It was $112(50.2 \%)$, $30(12.4 \%)$ and $35(14.5 \%)$ in the control group respectively. There was steady improvement in lifestyle score in the experimental group after intervention.

Ho: There is no significant difference between pre, post-test 1 and post-test 2 in the two groups.

Comparison of mean score of knowledge, attitude and lifestyle variables in groups: This table shows the mean scores of knowledge, attitude and lifestyle variables in experimental and control groups pre and post intervention. At baseline, mean scores of respondents on knowledge in Experimental and Control were 8.7 and 8.3 respectively (SD $8.49 \pm 4.03$ ). For attitude it was 46.3 and 51.3 respectively, (SD $45.10 \pm 6.51$ ) while for lifestyles it was 21.9 and 20.7 respectively, (SD $21.21 \pm 4.92$ ). At end-line, mean scores of respondents on knowledge in Experimental and Control were 15.6 and 5.7 respectively, (SD 5.22 \pm 5.22 ). For attitude it was 44.2 and 41.2 respectively, (SD $45.75 \pm$ 7.35), while for lifestyles it was 21.9 and 20.7 respectively (SD 20.63 \pm 5.60 ). The mean scores of respondents on knowledge, attitude and lifestyle of Experimental and Control groups at the three phases with $P$ value $<0.05$ in Experimental group and $>0.05$ in the Control. There was significant difference between the knowledge, attitude and lifestyle scores of experimental and control, P value 0.000 (Table 5).

\section{Discussion}

Comparison of findings at the three phases showed significant difference between the scores of participants in the Experimental and Control group after intervention on knowledge, attitude, lifestyle $(p<0.05)$. Improvement was also noted in the attitude and lifestyle of the control though not significant $(\mathrm{P}>0.05)$. This may be attributed to the method of data collection which in its self is a form of intervention or hawthorn effect as observed in studies of this nature. It could also be due to other sources of information which is beyond the control of the researcher. Non responses, even though it did not affect the findings was observed more in the control which may be as a result of apathy observed among members. It was perceived that their expectation was that drugs would be dispensed even though they were informed there will be no payment. Prevalence of hypertension was on the increase. Only very few were obese. This may be because majority are farmers thus physically active. Fifty percent (50.0\%) of all the respondents had correct knowledge of hypertension at baseline. This was lower than the findings by Familoni and Olunuga in Benin City where $59.3 \%$ of the non-clinical workers and $63.2 \%$ of the factory workers in the study had correct knowledge of hypertension [16]. This difference may be attributed to the rural setting in this study. However there was improved knowledge in the experimental group in subsequent assessments. This change may be the influence of the health education received by the Experimental group. Respondents with tertiary education had the highest score in knowledge of hypertension (44\%). This is in line with the study by Samal et al. in which knowledge of risk factors was related to level of knowledge. Ninety percent knew that stroke is a complication of hypertension, $61.1 \%$ knew of negative impact of table salt on blood pressure as against $77 \%$ and $54 \%$ respectively [17]. Unlike the study by Familoni and Olunuga in which $50 \%$ knew treatment is for life, in this study it was lower [16]. There is need for dissemination of accurate information to the controls that hypertension can only be controlled and not cured.

\begin{tabular}{|c|c|c|c|c|}
\hline Variable & Group & Period & Mean \pm SD & P-Value \\
\hline \multirow[t]{8}{*}{ Knowledge } & Experimental & & & \\
\hline & & Baseline & $8.67 \pm 3.92$ & \\
\hline & & Midterm & $15.30 \pm 0.99$ & 0.000 \\
\hline & & End-line & $15.64 \pm 0.52$ & \\
\hline & Control & & & \\
\hline & & Baseline & $8.35 \pm 4.13$ & \\
\hline & & Midterm & $6.09 \pm 2.62$ & 0.421 \\
\hline & & End-line & $5.69 \pm 2.15$ & \\
\hline \multirow[t]{8}{*}{ Attitude } & Experimental & & & \\
\hline & & Baseline & $46.25 \pm 4.75$ & \\
\hline & & Midterm & $62.67 \pm 5.88$ & 0.000 \\
\hline & & End-line & $51.28 \pm 6.22$ & \\
\hline & Control & & & \\
\hline & & Baseline & $44.40 \pm 7.55$ & \\
\hline & & Midterm & $43.36 \pm 9.32$ & 0.826 \\
\hline & & End-line & $41.21 \pm 4.57$ & \\
\hline \multirow[t]{8}{*}{ Lifestyle } & Experimental & & & \\
\hline & & Baseline & $21.89 \pm 4.53$ & \\
\hline & & Midterm & $17.00 \pm 2.19$ & 0.000 \\
\hline & & End-line & $26.17 \pm 1.89$ & \\
\hline & Control & & & \\
\hline & & Baseline & $20.65 \pm 5.17$ & \\
\hline & & Midterm & $8.85 \pm 2.40$ & 0.666 \\
\hline & & End-line & $16.17 \pm 3.02$ & \\
\hline
\end{tabular}

Table 5: Mean scores of respondents on knowledge, attitude and lifestyle within groups. 
Majority of the respondents with negative attitude to hypertension were of middle age. This age group should be targeted in programmes for behavior change. Many respondents strongly disagreed to having hypertension in their lifetime, whereas only $30 \%$ considered themselves at increased risk of stroke [17]. In this study fifty percent believed that the ailment is not for young people despite the fact that hypertension in younger people, especially before the age of 30 is usually more fatal than in the late fifties [18]. Same belief was similar to the participants in the Ibadan study in which $93 \%$ had difficulties to accept having new/ additional Non Communicable Disease (among which hypertension is one) [19]. Apparently these participants may not go for routine blood pressure check based on their belief. However the negative attitude in this study was lower than that of the Ibadan study.

Twenty four percent strongly agreed that hypertension affects women more. Thirty percent of the participants believe that irrespective of adoption of a healthy lifestyle hypertension will still occur. This implies that some participants will readily adopt prescribed healthy lifestyle while some would not. The percentage of respondents' with positive attitude improved from $60 \%$ to $88 \%$ after intervention in the experimental group. The positive change in attitude may be attributed to the perception of hypertension as a serious ailment or peer influence during 'walking exercise' as observed by researcher. In this study, $55 \%$ have similar believe as participants in the study by Busari et al., in which $53.8 \%$ believed that the goal of treatment was to cure the disease [20]. The fact that hypertension can only be controlled need to be emphasized by Community Health practitioners to limit incidence of stroke. Even though statistically there was no association between age, sex and level of knowledge, it was observed from the findings that positive attitude was more marked in female (58\%) than in males $(42 \%)$. This may be due to a culture where men have all the authority as head of the family. Women are house bound in the evenings, with men gathering in their social circles, encouraging unhealthy lifestyle habits as goat head, and cow tail and alcohol consumption amongst cohorts. Negative attitude was also least marked among the respondents with no formal education, which may be due to lack of influence of Western culture which makes one more assertive. Women must be empowered so that they can influence their men's attitude. The unhealthy lifestyles in relation to nutrition include chewing of kolanut, use of table salt, alcohol and cigarette. These risk factors were also identified by Aghaji and Omorogiwa, et al., in their studies [21,22]. Consumption of snuff by some respondents was identified in this study. This may be because rural dwellers are mainly old people who prefer snuff to cigarette. A minor percentage of participants were obese. This may be because majority are peasant farmers. Majority of the respondents in this study have witnessed stroke victims in their community and therefore perceived hypertension as a serious ailment. This experience may have also contributed to eagerness in the experimental group to know remedies for prevention and control of ailment which led to compliance to prescribed regimes that resulted in the improvement in lifestyle habits after intervention. Sixty (60\%) include vegetable in their meal always in experimental group and $54.1 \%$ in control. The liberal use of vegetable may be rooted in the Ibo culture of adding vegetable to all soup. This may have also contributed to the non significant difference in nutrition score of experimental and control after intervention. Food choices did not constitute a problem to the participants as was the study in China, in which lack of healthy food choices were their major barriers to lifestyle modification [23]. Lifestyle habits improved positively from $60 \%$ at baseline to $98 \%$ midterm and $100 \%$ at end-line in the Experimental group. The relapse which occurs at the maintenance stage of the transtheoretical model of behavior change was slightly noted. This may likely be due to the flier on healthy lifestyles, the role play which must have served as 'reminders' and support from significant others since the ailment was perceived as serious. This emphasizes on the importance of follow up and reinforcement in behavior modification or change. Regular follow up, even after this study should be maintained in the experimental community for the behavior change to be sustained and same intervention should also be replicated in the control community.

\section{Conclusion}

There was marked improvement in knowledge, attitude and lifestyle relating to hypertension control after community health nursing intervention in Isunjaba. This led to improved regular blood pressure checks by community members, donation of blood pressure measuring tools by philanthropists for distribution in all wards. Regular health promotion information by nurses is recommended for sustainability of knowledge, attitude, health promoting activities and lifestyle. Study need to be replicated in other rural communities in Nigeria to arrest the upward trend in hypertension prevalence in the country and Africa sub-region.

Greater percentage of the respondents had low knowledge at baseline but knowledge improved greatly in the experimental group after intervention. More than half of the participants with high blood pressure were only revealed during screening. Knowledge and awareness of risk factors is a component of behavior change as shown by finding in this study, Self-measurement blood pressure devices should be possessed by each household. Mobile clinics involving collaborative approach should be adopted. Policy on attitudinal and lifestyle changes in relation to hypertension prevention and control amongst the youth and middle aged should be put in place. Even though $70 \%$ agreed that hypertension is a serious ailment, only half of the participants have positive attitude and were willing to take up recommended lifestyle habits. Programme for attitudinal change need to be intensified for the youth and middle aged amongst whom the negative attitude and poor lifestyle were most marked. Continuity of the programme is important for sustainability. The intervention programme should also be carried out in the Control community as well.

\section{Acknowledgements}

Dr O Abimbola Oluwatosin and Prof $S$ Kadiri of the Faculty of Clinical Sciences, University of Ibadan, Oyo State, Nigeria are being acknowledged for their supervision of this study.

\section{References}

1. Jolly S, Vittinghoff E, Chattopadhyay A, Bibbins-Domingo K (2010) Higher cardiovascular disease prevalence and mortality among younger blacks compared to whites. The American Journal of Medicine 123: 811-818.

2. Okpechi IG, Chukwuonye II, Tiffin N, Madukwe OO, Onyeonoro UU, et al. (2013) Blood pressure gradients and cardiovascular risk factors in urban and rural populations in Abia State South Eastern Nigeria using the WHO STEP wise approach. PLoS ONE 8: e73403.

3. World Health Organization (2002) The world health report 2002: reducing risks, promoting healthy life. World Health Organization.

4. Das SK, Sanyal K, Basu A (2005) Study of urban community survey in India: growing trend of high prevalence of hypertension in a developing country. Int J Med Sci 2: 70-78.

5. Abonnema Foundation (2013) Hypertension in Nigeria: Rising to the challenge

6. Metintas S, Arikan I, Kalyoncu C (2009) Awareness of hypertension and other cardiovascular risk factors in rural and urban areas in Turkey. Transactions of the Royal Society of Tropical Medicine and Hygiene 103: 812-818.

7. Egan BM, Zhao Y, Axon RN (2010) US trends in prevalence, awareness, treatment, and control of hypertension, 1988-2008. JAMA 303: 2043-2050.

8. Iyalomhe GB, lyalomhe SI (2010) Hypertension-related knowledge, attitudes and life-style practices among hypertensive patients in a sub-urban Nigerian community. Journal of Public Health and Epidemiology 2: 71-77. 
Citation: Osuala Eunice O (2017) Hypertension Prevention and Control: Effects of a Community Health Nurse-led Intervention. J Health Educ Res Dev 5: 210. doi: 10.4172/2380-5439.1000210

9. Ekwunife OI, Udeogaranya PO, Nwatu IL (2010) Prevalence, awareness, treatment and control of hypertension in a Nigerian population. Health 2: 731735.

10. Osuala EO, Abimbola OO, Kadiri S (2014) Knowledge, attitude to hypertension and lifestyle habits of rural dwellers in Owerre-Nkwoji, Imo State Nigeria. Journal of Public Health and Epidemiology 6: 48-51.

11. Demaio AR, Otgontuya D, de Courten M, Bygbjerg IC, Enkhtuya P, et al. (2013) Hypertension and hypertension-related disease in mongolia; findings of a national knowledge, attitudes and practices study. BMC Public Health 13: 194.

12. Oladapo OO, Salako L, Sadiq L, Soyinka K, Falase AO (2013) Knowledge of hypertension and other risk factors for heart disease among Yoruba rural South Western Nigeria. BJMMR 4: 993-1003.

13. Balogun MO, Olugbenga O (2013) Prevalence of hypertension in three rura communities of Ife north local Government Area of Osun state, south West Nigeria. International Journal of General Medicine 6: 863-868.

14. Maxwell FP (1998) A-Z of Medical statistics. A comparison for critical appraisal. Oxford University Press Inc., New York, USA.

15. US Bureau (2006) United States Census Bureau. Household Income.

16. Familoni OB, Olunuga TO (2005) Comparison in the knowledge and awareness of hypertension among hospital and factory workers in Sagamu, Nigeria. Nigerian Medical Practitioner 47: 43-45.
17. Samal D, Greisenegger S, Auff E, Lang W, Lalouschek W (2007) The relation between knowledge about hypertension and education in hospitalized patients with stroke in Vienna. Stroke 38: 1304-1308.

18. Ige OK, Owoaje ET, Adebiyi OA (2013) Non communicable disease and risky behavior in an urban University Community in Nigeria. Afr Hlth Sci 13: 62-67.

19. Busari OA, Olusegun T, Olufemi O, Desalu O, Opadijo OG, et al. (2010) Impact of Patients' Knowledge, Attitude and Practices on hypertension on compliance with antihypertensive drugs in a resource-poor setting. TAF Prev Med Bull 9: 87-92.

20. Aghaji MN (2008) Hypertension and risk factors among traders in Enugu, Nigeria. Journal of College of Medicine 13: 114-115.

21. Omorogiwa A, Ezenwa EB, Osifo C, Ozor MOE, Khator CN (2009) Comparative study on risk factors for hypertension in University setting in Southern Nigeria. International Journal of Biomedical and Health Sciences 5: 103-107.

22. Chan RSM, Lok KYW, Sea MM, Woo J (2009) Clients' experiences of a commodity based lifestyle modification programme: A qualitative study. Int $\mathrm{J}$ Environ Res Public Health 6: 2608-2622.

23. Prochaska JO, Redding CA, Evers KE (2002) The Transtheoretical Model and Stages of Change in Health Behaviours and Health Education: Theory, Research and Practice. New Jersey, USA, pp: 280-282. 